\title{
Case Report of Idiopathic Sialectasis of Stensen's Duct and Literature Review
}

\author{
Chandira Gunasena ${ }^{1}$, Gamini Nawarathne ${ }^{2}$, Ananda Rathnayake ${ }^{3}$, Chandima Weerasinghe ${ }^{4}$
}

\begin{abstract}
This paper discusses a case of idiopathic sialectasis of the Stensen's duct in a 51-year-old male patient diagnosed and managed successfully. Idiopathic sialectasis is a rare presentation. Diagnosis of this entity is by clinical, radiological, and ultrasonological correlation. The literature on this entity is scarce, so this paper intends to provide a literature review and analysis into the epidemiology and management aspects.

Keywords: Case report, Idiopathic, Sialectasis, Stensen's duct.

Journal of Oral Health and Community Dentistry (2021): 10.5005/jp-journals-10062-0092
\end{abstract}

\section{INTRODUCTION}

Sialectasis is defined as dilatation of a salivary duct. It is an uncommon phenomenon manifested in adults. ${ }^{1,2}$ It usually manifests in the parotid gland and submandibular gland. ${ }^{2}$ Etiology for this disease entity can be due to salivary duct obstruction due to sialoliths, salivary mucus plug formation, strictures of duct, and stenosis of duct, recently accepted that there is an involvement due to the masseter muscle and idiopathic. ${ }^{2,3}$

Ductal sialectasis clinically appears as increasing cheek swelling, next proceeding with pain, and facial asymmetry. ${ }^{2,3}$ They are not aggravated by food but relieved by massaging the lesion. ${ }^{3}$ On examination, they appear as diffuse swellings with firm sensation and slightly mobile. ${ }^{3,4}$ On applying pressure on the lesion, there will be evacuation of saliva from the parotid duct. ${ }^{7}$ These can be confirmed by sialography, ultrasonography (US), magnetic resonance imaging (MRI), computed tomography (CT), and sialendoscopy. ${ }^{7,8}$

Treatment mainly consists of conservative and surgical methods. ${ }^{7}$ Conservative modalities include repeated aspiration, compression, dilatation of the papilla, and stent placement. ${ }^{7}$ Surgically, there is a bypass by stent placement from the point of dilatation and parotidectomy. ${ }^{7}$

Presentation of idiopathic sialectasis is rare. ${ }^{7}$ Due to its rarity, there is a sparse amount of literature discussing the epidemiology and management of this entity. In this case, we discuss a modified method of treatment, combining both conservative and surgical approaches; also intend to elaborately review the epidemiology and management options mentioned in the literature.

\section{Case History}

A 51-year-old male presented to the Oral and Maxillofacial Unit A of Dental Institute, Colombo, with a complaint of swelling on the right side of the cheek of 4 months' duration. He stated it was a nonpainful swelling, causing facial asymmetry and increasing gradually. It was not aggravated due to the sensation of food or during eating. He relates no incidence in relation to this lesion. This swelling is reducing when he massages it but it causes pain, so he refrains from it. He had received earlier treatment which did not resolve this condition. He had no medical problems or any drug
${ }^{1-4}$ Oral and Maxillofacial Unit A, Dental Institute, National Hospital Colombo, Colombo, Sri Lanka

Corresponding Address: Chandira Gunasena, Oral and Maxillofacial Unit A, Dental Institute, National Hospital Colombo, Colombo, Sri Lanka, Phone: +0776120616, e-mail: cgunasena039@gmail.com

How to cite this article: Gunasena C, Nawarathne G, Rathnayake A, et al. Case Report of Idiopathic Sialectasis of Stensen's Duct and Literature Review. J Oral Health Comm Dent 2021;15(1):37-40.

Source of support: Nil

Conflict of interest: None

allergies or food allergies. He had no trauma to that region or any past surgical history. He has had several extractions. He works as a security guard for a private company.

Generally, the patient appeared healthy and there were no abnormalities. Extraorally, he had diffuse swelling on the right side of the cheek region in the parotid duct area, measuring about $3 \times 3 \mathrm{~cm}$ (Fig. 1). It was slightly firm and mobile. There was no sensation of parotid calculi on bimanual palpation of the parotid duct area.

Intraorally, the swelling extended up to the buccal vestibule but did not cross it (Fig. 2). The patient had generalized gingivitis, caries in 27 , and the remaining root in 26 . The patient was partially dentate.

Dental panoramic tomography (DPT) revealed there was generalized bone loss, pulp exposed 27, and retained root of 26 . There was no pathology located on the right side (Fig. 3).

Differential diagnosis of sialocele, parotid duct dilatation, and dermoid cyst was considered.

To investigate further, ultrasound scan of that region was prescribed. Sialography was not considered as the swelling was significant and might cause pain to the patient during the injection of contrast media. Ultrasound revealed a cyst-like swelling due to distal parotid dilatation. A final diagnosis of sialectasis was made (Fig. 4).

Under local anesthesia, a corrugated rubber dam of $2 \mathrm{~mm}$ in length was placed after an incision was made until the parotid duct after locating the dilatation. Pressure was applied around the swelling, forcing the expulsion of saliva. The patient was prescribed sialagogues, like vitamin C, to chew four times a day and analgesics. The patient was advised to massage the swelling three times a day and to be reviewed in 2 weeks. 


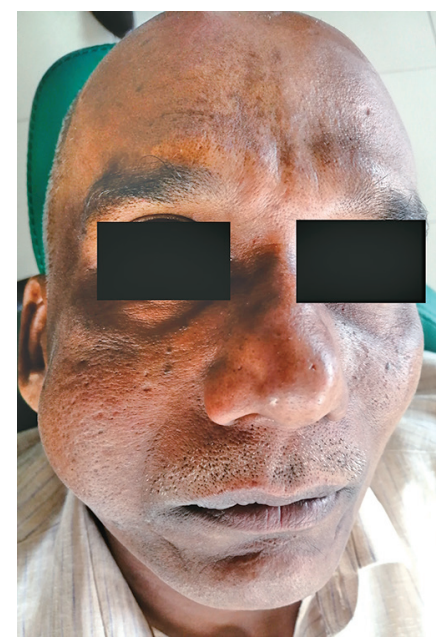

Fig. 1: Photograph of the patient in first visit

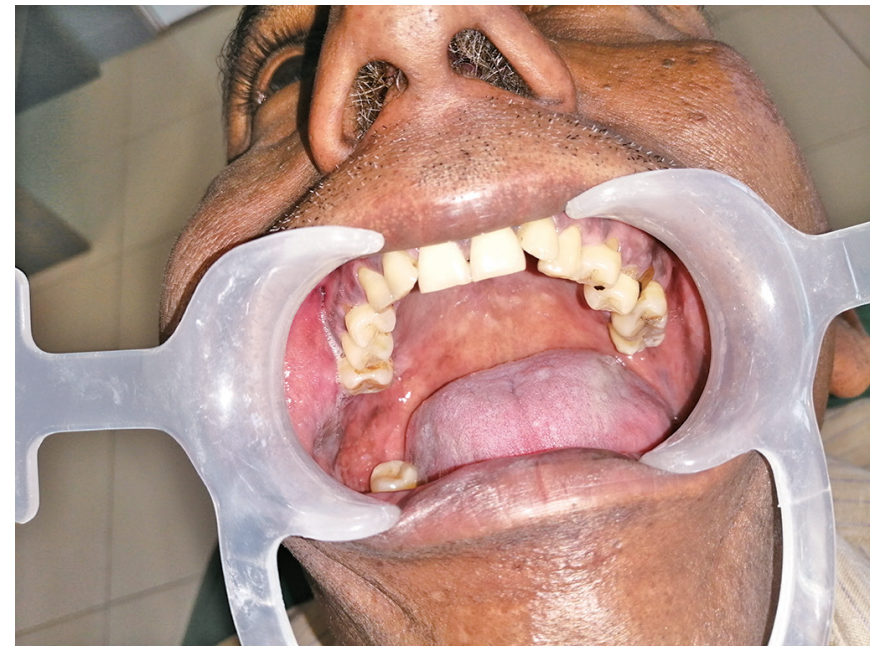

Fig. 2: Intraoral photograph at initial presentation

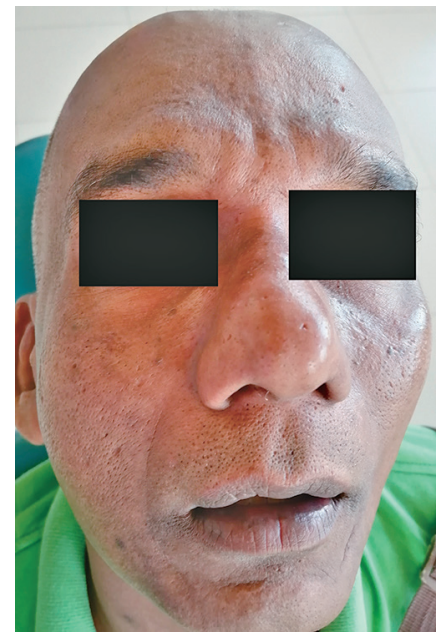

Fig. 3: Photograph of the patient in "review after surgery"

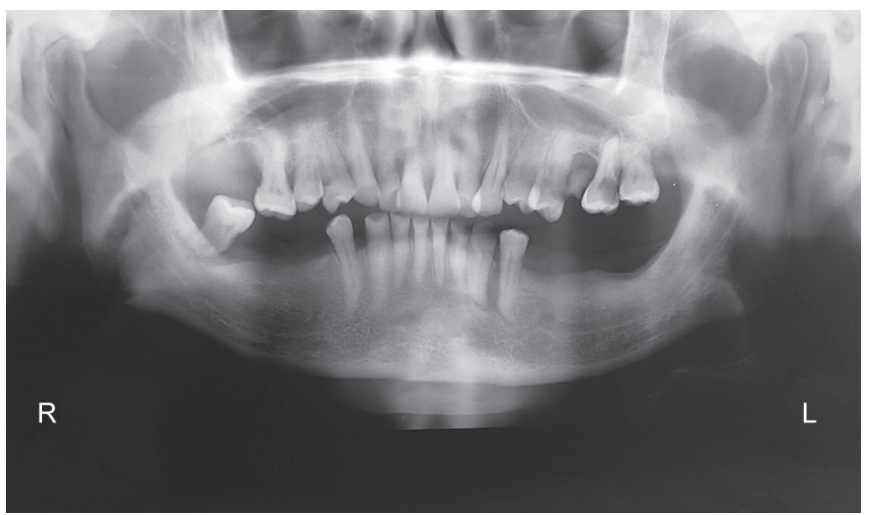

Fig. 4: Dental panoramic tomograph of the patient

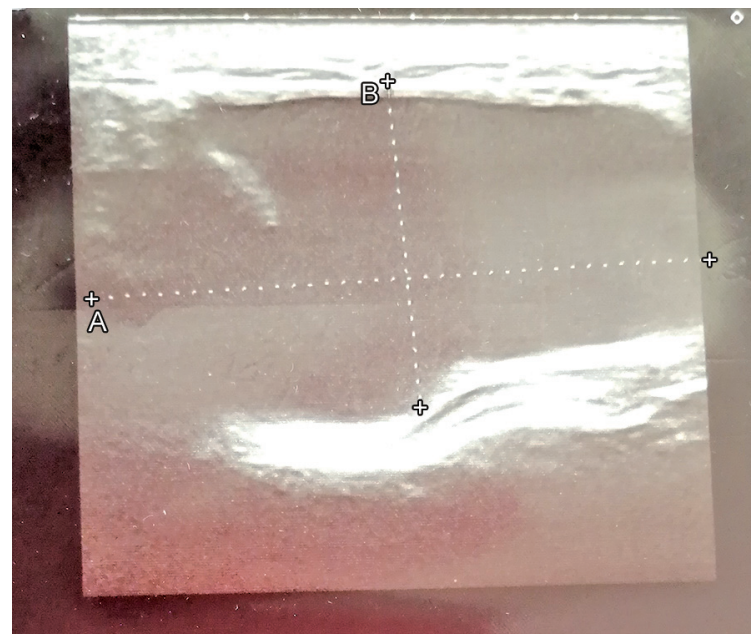

Fig. 5: Ultrasound scan image of the sialectasis

In the 2-week review, the swelling completely revolved (Fig. 5). The rubber dam was removed and the patient was referred for other treatment.

\section{Discussion}

Merriam-Webster dictionary defines sialectasis as abnormal dilatation of a duct in the salivary gland. ${ }^{3}$ Sialectasis is an uncommon phenomenon. It is caused due to obstructions in the salivary ducts, ductal stenosis, foreign bodies, and anatomical anomalies in the duct, and is idiopathic., ${ }^{3,4}$ Obstructions can be due to sialoliths and intraductal papilloma. The occurrence of sialoliths is common in the submandibular gland (80-90\%), seconded by the parotid gland (5-15\%), followed by the sublingual gland (2-5\%). ${ }^{1}$ Obstruction of the Stensen's duct is uncommon. The incidence of intraductal papilloma in Stensen's is rare as ductal papillomas are common in minor salivary glands. ${ }^{14}$ Ductal stenosis occurs due to secondary causes, such as following sialolithectomy, traumatic ductal injury due to assault or surgery, and longstanding chronic parotitis, causing fibrosis of the duct. ${ }^{7}$ Familial involvement was also documented by Smith but is not well established in the literature. ${ }^{6}$ There is also the emergence of an idea for the etiology, stating that masseteric hypertrophy is a contributory factor. ${ }^{7}$ Idiopathic focal sialectasis is rare in 
Case Report of Idiopathic Sialectasis of Stensen's Duct

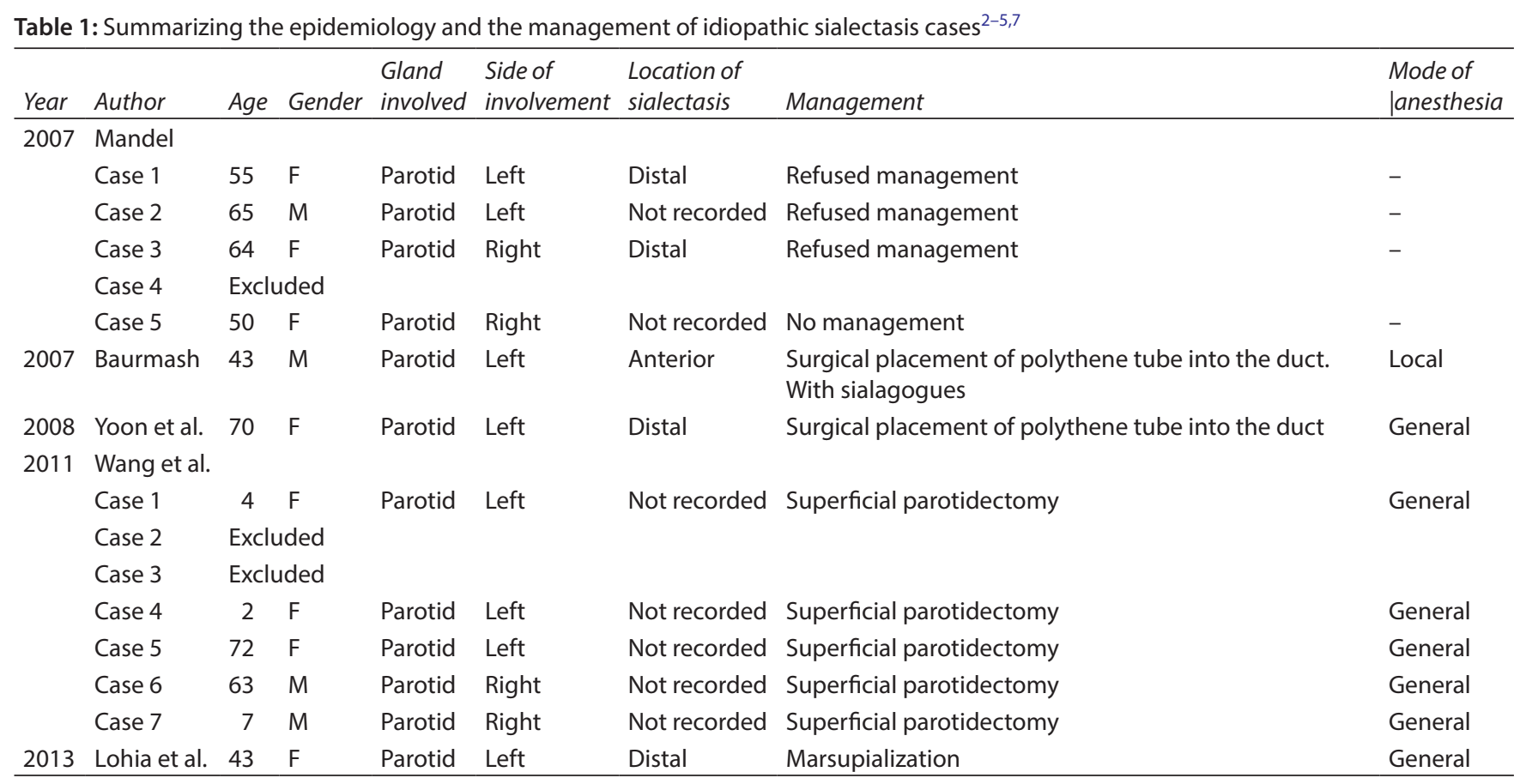

presentation. ${ }^{7}$ The prevalence of this entity is not documented in the literature; literature that exists is mainly a few researches, case reports, and case series. The etiology of our case is idiopathic as the clinical history does not correspond to any of the mentioned etiologies.

Table 1 summarizing the literature published from 2000 up to date regarding idiopathic sialectasis confirmed by clinical, radiological, and ultrasonological criteria and the literature published in English.

When we consider idiopathic sialectasis, epidemiology is not well documented in the literature. According to Table 1, the age ranges from 2 to 70 years and the mean age is 44.8 years. This entity has a slight female gender disposition, in which the female to male ratio is 1.75:1. When we consider the present case, it lies in the age range and above the mean age.

All of these cases summarized in Table 1 involve the parotid gland so is the present case. The most commonly involved side is the left side, where the ratio of left to right side parotid glands is 2:1. In our case, a right-sided involvement is seen. Bilateral presentation is not observed in the literature. Submandibular involvement is not mentioned in the literature.

Clinically, this entity can be longstanding, asymptomatic swelling manifested in the cheek. There can be episodes of pain when the swelling becomes significantly large. There are no exaggerating factors like stimulation due to meals. There are alleviating factors like massaging the swelling which is due to the expulsion of saliva from the duct. These features correspond with the present case.

On examination, this entity will give the appearance of a diffuse cheek swelling, which is seen both extraorally and intraorally. It is firm in palpation clinically and mobile. It can vary in size. Dilation of the parotid duct depends upon the severity of the obstruction, elasticity of the duct, and glandular function. ${ }^{3}$ According to Table 1, distal point dilation is more prevalent than anterior dilation with a ratio of 4:1 (distal:anterior dilation).
Other differential diagnoses of this presentation consist of angioedema, lipoma, pneumoparotid, odontogenic infection, and sialocele.

Angioedema is an immune-mediated condition which consists of a cell-mediated angioedema and bradykinin-mediated angioedema. Salient features of this entity are erythema of the skin and urticaria with swelling involving bilateral aspects due to a precipitating factor. Hereditary angioedema occurs without any predisposing factor. ${ }^{13}$ Based on the clinical evidence of the present case, angioma was excluded.

Lipomas are benign mesenchymal tumors occurring in the body. It occurs in the arm, shoulder, back, legs, forehead, and face. ${ }^{12}$ Presentation in the face is uncommon, it occurs in regions, such as the cheek followed by tongue, buccal mucosa, lip, and neck. In the present case, the possibility of a lipoma was considered but was excluded based on the clinical evidence. ${ }^{12}$

Pneumoparotid is defined as a pathological entity of entry of air into the parotid gland. It is a very rare phenomenon. It is due to the development of large intraoral pressure. It presents clinically as an asymptomatic swelling in the cheek which is similar to the present case. There is crepitus on palpation of the gland, and frothy saliva or air bubbles may be observed, emanating from the Stensen's duct during massage of the gland on further examination, so this diagnosis was excluded based on the clinical evidence in the present case. ${ }^{15}$

Odontogenic infections were excluded clinically and radiographically in our case.

Sialocele is a subcutaneous cavity containing saliva, usually results from a trauma or an infection to the parotid gland, laceration of the parotid duct, or ductal stenosis with subsequent dilatation. Clinically, features had similarities with the present case but the history of the present case contraindicates this entity. Ultrasonologically also features were not in favor of this lesion. ${ }^{16}$

The various imaging modalities to investigate salivary gland disorders include conventional radiography, sialography, US, CT, 
radionuclide imaging, and MRI. Sialography is one of the oldest imaging procedures, as it is a simple chairside procedure, easy to perform, and cost-worthy. Sialectasis is the sialographic appearance of dots or blobs of contrast medium within the gland caused by inflammation of the glandular tissue, producing saccular dilatation of the acini. Sialography is the choice of radiographic examination of the salivary glands. It usually involves injection of a small amount of contrast medium into the salivary duct of a single gland followed by routine X-ray projections. To diagnose diseases of the salivary glands and for staging of the disease and treatment planning, detailed imaging is always necessary. ${ }^{8}$ In the present case, a preoperative sialogram could not be attempted due to pain, which can be inflicted due to the large swelling. Ultrasound scan provides more details of any salivary pathology with great accuracy and it is not invasive and comfortable for the patient as well. ${ }^{10,11}$

Sialendoscopy is a procedure done by inserting a miniaturized flexible endoscope in the salivary ducts for diagnostic and treatment purposes. It is commonly used for removal of sialoliths and treatment of ductal strictures by introducing balloons. ${ }^{9}$

Management of this entity consists of conservative and surgical approaches. The conservative approach consists of repeated aspiration, compression, dilation of the papilla, and stent placement. They do not always give a predictable success rate. In case of failure, surgical management should be looked upon. ${ }^{7}$

Surgical methods consist of excision of the dilated ductal part and placement of a stent, ductal bypass, placement of tubes by an incision into the parotid duct, and superficial parotidectomy. ${ }^{7}$ These methods give predictable success. Tube placement with an incision into the parotid gland gives a good success as described by Baurmach and Yoon et al. This is the same method we followed in our case. The basis behind our method was to introduce an artificial path distal to the sialectasis for the saliva to be removed from the parotid gland long enough to create a sinus tract, thus producing another salivary ductal opening.

Lohia et al. had done marsupialization of the duct under general anesthesia and connected it to the buccal mucosa. ${ }^{7}$ He chose that method because of the ductal stenosis found in the preoperative sialendoscopy. It produced good results. Sialendoscopy was not attempted in our case as that facility was not available.

Wang et al. had treated seven cases of congenital sialectasis with superficial parotidectomy with effective results. ${ }^{5}$ The risks of superficial parotidectomy will be very invasive and sialocele formation. This option was considered in the present case as the last resort but did not need, as the first treatment method worked. When we consider the management in the present case, it is a slightly invasive, blind, and cost-effective method which gives good results. This modality can be used as the first-line mode of surgical treatment based on our experience.

Based on evidence from the literature we have cited here, it shows that surgical methods have good results. Further inquiry into this aspect is needed.

There is a limitation to the literature analysis in Table 1. All literatures published before 2000 were not included. So, this attempt is a preliminary effort to understand the epidemiology of this entity. Further review is advisable to understand this entity deeply.

\section{Conclusion}

Idiopathic sialectasis is a rare presentation in adults. Diagnosis by exclusion by other conditions will spare unnecessary management. US is a noninvasive but an effective mode of diagnosis, especially locating the point of sialectasis. Surgical management combined with other supplementary managements will give good results quickly.

\section{ACKNOWLEDgMentS}

Authors would like to acknowledge the staff of OMF Unit A, Dental Institute, Colombo, and the patient.

\section{References}

1. Greenberg MS, Glick M. Burket's oral medicine. 10th ed. Elsevier Publication; 2003. p. 238-239.

2. Mandel L. The grossly dilated Stensen's duct: case reports. J Oral Maxillofac Surg 2007;65(10):2089-2094. DOI: 10.1016/j.joms.2006. 03.046 .

3. Baurmash HD. Sialectasis of Stensen's duct with an extraoral swelling: a case report with surgical management. J Oral Maxillofac Surg 2007;65:140-143. DOI:10.1016/j.joms.2005.12.033.

4. Yoon YH, Rha KS, Choi JW, et al. Sialectasis of Stensen's duct: an unusual cause of recurrent cheek swelling. Eur Arch Otorhinolaryngol 2009;266(4):573-576. DOI: 10.1007/s00405-008-0702-0.

5. Wang $Y, Y u G Y$, Huang MX, et al. Diagnosis and treatment of congenital dilatation of Stensen's duct. CORD Conf Proc 2011;121(8):1682-1686. DOI: 10.1002/lary.21854.

6. Smith M. Medical Memorandum. Br Med J December 1953.

7. Lohia S, Joshi AS. BMJ Case Rep 2013;2013. DOI: 10.1136/ bcr-2013201548.

8. Metha SS, Mhapuskar AA, Hiremutt D, et al. Chronic parotid sialadenitis with sialectasis: diagnosis of case through $C T$ sialography. J Pharm Biomed Sci 2016;06(04): 234-237.

9. Singh PP, Gupta V. Sialendoscopy: introduction, indications and technique. Indian J Otolaryngol Head Neck Surg 2014;66(1):74-78. DOI: 10.1007/s12070-013-0675-1.

10. Bialek EJ, Jakubowski W, Zajkowski P, et al. US of the major salivary glands: anatomy and spatial relationships, pathologic conditions, and pitfalls. Education exhibit. RadioGraphics 2006;26(3):745-763. DOI: 10.1148/rg.263055024.

11. Quigley AJ, Kulkarni S, McAteer GLD. Sialography—a technique worth revisiting: normalanatomy, anatomical variants and duct pathology Educational Exhibit ECR 2014. C-2165. DOI: 10.1594/ecr2014/C-2165.

12. Derin AT, Yaprak N. Lipomas: review and evaluatıon of the literature. Review article. Clin Surg 2017;2:16151.

13. Nedelea I, Deleanu D. Isolated angioedema: an overview of clinical features and etiology. Exp Ther Med 2019;17(2):1068-1072. DOI: 10.3892/.2018.6982.

14. Brannon RB, Sciubba JJ, Giulani M. Ductal papillomas of salivary origin. Report of 19 cases and review of literature. Oral Surg Oral Med Oral Pathol Oral Radiol Endod 2001;92(1):68-77. DOI: 10.1067/ moe.2001.115978.

15. Lee GG, Lee J, Kim BY, et al. A case of pneumoparotid: initially presented with viral parotitis. Korean J Otorhinolaryngol-Head Neck Surg 2012;55:721-723. DOI: 10.3342/kjorl-hns.2012.55.11.721.

16. Araujo MR, Centurion BS, Albuquerque DF, et al. Management of a parotid sialocelein a young patient: case report and literature review. J Appl Oral Sci 2010;18(4):432-436. DOI: 10.1590/S167877572010000400019. 